Animal Genetics,

1999, 30, 190-194

\title{
Diagnostic value of a microsatellite DNA marker for copper toxicosis in West-European Bedlington terriers and incidence of the disease
}

\author{
J Rothuizen, GJ Ubbink, P van Zon, E Teske, TSGAM van den Ingh, \\ V Yuzbasiyan-Gurkan
}

\section{Summary}

Recently, linkage of a DNA microsatellite marker to inherited copper toxicosis has been reported in American Bedlington terrier families. Due to the fact that there is little exchange of breeding stock between the USA and Europe, it remains to be investigated whether in Europe the marker is informative and is linked with the disease. We have therefore examined the diagnostic value of the microsatellite marker in the European Bedlington. In 130 dogs at least one year of age (62 from The Netherlands, 35 from Belgium, and 33 from Germany) histo- or cytochemical staining of copper was done in liver biopsies. Based on liver histo- or cytochemistry, 51 dogs were obligate carriers, and 25 dogs had copper toxicosis. The inferred genotypes of these 76 dogs were compared with the marker genotypes. All dogs with the disease were homozygous for the $167 \mathrm{bp}$ marker allele. All obligate carriers were heterozygotes with the $167 \mathrm{bp}$ and a 163-bp alleles. All phenotypically healthy dogs were either homozygous for the $163 \mathrm{bp}$ allele or heterozygous. Thus, the marker was in complete linkage disequilibrium with the putative copper toxicosis gene with the $167 \mathrm{bp}$ allele in phase with the disease allele. The frequencies of the $167 \mathrm{bp}$ and the $163 \mathrm{bp}$ allele, respectively, were 0.33 and 0.67 in Dutch dogs, 0.31 and 0.69 in German dogs, and 0.57 and 0.43 in Belgian dogs. We have confirmed the utility of this marker for diagnosis of inherited copper toxicosis in European Bedlington terriers.

Keywords: Bedlington terrier, copper toxicosis, microsatellite, cytochemistry, DNA marker, incidence, Western Europe

Correspondence: J Rothuizen.

Accepted 5 January 1999

\section{Introduction}

Copper toxicosis is an inherited autosomal recessive disease in Bedlington terriers (Johnson et al. 1980; Owen \& Ludwig 1982). The disease is characterized by an inability of the hepatocytes to excrete the excess of copper absorbed in the intestinal tract into the bile. The result is a progressive accumulation of copper in the hepatocellular lysosomes which becomes distinguishable from the normal situation at one year of age (Hardy et al. 1975; Twedt et al. 1979; Herrtage et al. 1987). Clinical signs, usually at an age of five years or more (Hardy et al. 1975; Twedt et al. 1979) may be caused by the resulting chronic hepatitis eventually leading to cirrhosis, or by an acute and severe hemolytic crisis triggered by as yet unknown mechanisms. Although not all dogs become clinically ill, the disease is usually fatal. Only life-long treatment with drugs to prevent further accumulation of copper may prevent mortality (Twedt et al. 1988; Brewer et al. 1992).

Until recently, the diagnosis of copper toxicosis could only be made by measurement of copper in the liver, either quantitatively (Thornburg et al. 1990) or by semiquantitative histochemical or cytochemical detection (Johnson et al. 1984; Teske et al. 1992) at an age of at least one year. Unfortunately, only homozygous affected dogs can be recognized by copper measurements, and heterozygotes may only be detected when they have offspring or a parent with the disease. The incidence of the disease is about $25 \%$ in most countries (Herrtage et al. 1987; Yuzbasiyan-Gurkan et al. 1997), which implies that about two-thirds of the nonsymptomatic dogs must be carriers and onethird homozygous free of the disease. The disease has been reported in the USA (Hardy et al. 1975; Twedt et al. 1988), Australia (Robertson et al. 1983), and in several Western European countries (Eriksson 1983; Herrtage et al. 1987; Teske et al. 1992). Although with systematic evaluation of the copper status of breeding stock and their offspring, the incidence of the disease may be reduced (Ubbink et al., data not shown), this has proven effective 
191

Diagnostic value of a microsatellite DNA marker for copper toxicosis only to a certain extent because many heterozygotes cannot be discovered.

An anonymous microsatellite marker linked to the copper toxicosis gene has been reported in Bedlington terriers in the USA (YuzbasiyanGurkan et al. 1997). Although the American and the European Bedlington terrier population both originate from England, it is necessary to verify these findings before using the marker for diagnosis in European populations. For example, copper toxicosis in European Bedlingtons could be caused by a mutation in a different gene or the phase between the marker alleles and the disease alleles could be different.

We have examined the diagnostic value of the microsatellite marker reported by YuzbasiyanGurkan et al. (1997) in European Bedlington terriers.

\section{Materials and methods}

\section{Animals and samples for DNA isolation}

Bedlington terriers included in the study were at least one year of age. Four milliliters of blood was sampled by jugular venipuncture and collected in EDTA-coated tubes. At the same time, thin needle liver biopsies were taken for cytochemical quantitation of copper.

When we had enough material for analysis of the diagnostic value of the marker, no more liver tissue was sampled and samples were only taken for PCR of the marker locus. These samples were blood, brush swabs of the oral mucous membranes (Cyto-pak, Medical Packaging Corporation, Camarillo, CA), or roots of hair extracted with mosquito forceps. Different sources of hair from four dogs were compared: the head, the shoulder, the back, the inner side of the ears, and the lateral extremities.

Clinical data of copper measurements in histological liver biopsies from 155 recent ancestors of the present dogs that were examined before 1985 (Teske et al. 1992) were also employed. Together with the clinical data from the present study they were used to detect obligatory heterozygous carriers of the disease by combining relationships derived from the pedigrees and the presence of copper toxicosis.

\section{Liver samples and cytochemistry}

In all dogs liver tissue sampled by thin needle aspiration and the copper concentration was semiquantitatively assayed in the smears (Teske et al. 1992). In four dogs there was insufficient liver tissue in the smears and in these dogs a blind histological liver biopsy was taken under local anesthesia (Rothuizen 1998) and copper was measured histochemically (Teske et al. 1992).

\section{DNA isolation}

DNA was isolated from blood according to Miller et al. (1988). Hair roots were clipped off and treated with $50 \mu \mathrm{l}$ lysis buffer and $200 \mathrm{~g}$ proteinase $\mathrm{K}$ at $56{ }^{\circ} \mathrm{C}$ for $12 \mathrm{~h}$. After heat inactivation of the proteinase, $1 \mu \mathrm{l}$ of the supernatant was used in the PCR.

\section{Microsatellite PCR and electrophoresis}

The PCR was performed in $10 \mu \mathrm{l}$ reactions with $20 \mathrm{ng}(1 \mu \mathrm{l})$ of DNA as template as described by Yuzbasiyan-Gurkan et al. (1997); the reverse primer was labelled with $\gamma^{3}{ }^{32} \mathrm{P}$. After the PCR $30 \mu \mathrm{l}$ of loading dye containing formamide was added to each tube. Three microliters of the products were heated at $100{ }^{\circ} \mathrm{C}$ for $5 \mathrm{~min}$, immediately placed in melting ice, and then electrophoresed in a $6 \%$ polyacrylamide gel in $0.6 \times$ TBE and $8 \mathrm{~m}$ urea for $1.5 \mathrm{~h}$ at $60 \mathrm{~W}$ $(2000 \mathrm{~V})$. A molecular weight standard was loaded in a separate slot. A sensitive film was exposed to the gels for $12-24 \mathrm{~h}$ after electrophoresis. The length of the PCR products was determined for 20 dogs using a fluorescent instead of a radioactive primer, and analysis of the products on an ABI-310 Genetic Analyser (Perkin Elmer, Nieuwerkerk aan de IJssel, The Netherlands).

\section{Data analysis}

The diagnostic value of the results of the microsatellite analysis was analysed by comparing it with the independently assessed genotype based on the results of the histo- or cytochemical liver analysis of copper in the liver, and pedigree analysis. The genotypes of dogs with clinical disease and of the obligatory carriers were used as the independent 'golden' standard. The accuracy of the results of microsatellite analysis was calculated as the fraction of agreement with the golden standard, and the upper and lower 95\% confidence intervals were calculated based on the number of comparisons, according to Zarr (1984).

\section{Results}

Liver biopsies and blood samples for microsatellite analysis were obtained from 130 dogs: 62 from The Netherlands, 35 from Belgium, and 
192

Rothuizen, Ubbink, van Zon et al.
33 from Germany. Twenty-five of them had pathological copper levels in their liver, whereas the others had normal copper levels and no evidence of liver pathology. Using these and results from previous studies (Teske et al. 1992, Ubbink et al. unpublished data) and pedigree records, we could identify 51 obligate heterozygous carriers. Thus, the genotype of 76 dogs could be inferred with certainty from the phenotypic data.

In these 130 dogs, microsatellite analysis revealed two alleles; one of $163 \mathrm{bp}$ and the other $167 \mathrm{bp}$ in length. All dogs with pathological copper levels in their livers were homozygous for the $167 \mathrm{bp}$ allele, and all 51 obligate carriers were heterozygous. All phenotypically healthy dogs whose genotype could be inferred from their pedigree records were heterozygous or 163 bp homozygotes. Thus, we found $100 \%$ agreement between the two tests, and with the number of dogs studied the diagnostic accuracy of the test was between 95\% and 100\% (95\% confidence intervals).

After having found that the diagnostic accuracy of the DNA marker was at least $95 \%$, more dogs of the populations were analysed without further comparisons with liver cytochemistry. In some of these cases DNA was derived from blood samples, but in most cases owners were requested to send samples of mouth swabs or hairs by post. Hairs from the back (both the whither and the buttock) always gave a good yield of usable DNA while DNA yield and quality from hairs from other regions were more variable, not always giving detectable PCR products. Therefore only hairs extracted from the buttock were employed. The mouth brush swabs gave much lower quantities or quality of DNA than hair.

The results of the microsatellite analysis on a total of 290 dogs are summarized in Table 1. For all three populations the expected distribution of genotypes assuming Hardy-Weinberg equilibrium agreed very well with the distribution actually found.

\section{Discussion}

The present study has revealed that the DNA marker for copper toxicosis identified by Yuzbasiyan-Gurkan et al. (1997) provides an accurate diagnostic test in Bedlington terriers of the Western-European continent, and that the overall-frequency of the marker allele associated with copper toxicosis is $37 \%$.

We have used histo- and cytological analyses of liver tissue as the basis to define a 'golden' standard for evaluating the accuracy of the marker test. We only used dogs at least one year old, for the phenotype is always apparent in dogs of that age and beyond (Hardy et al. 1975; Twedt et al. 1979; Teske et al. 1992). To reduce any misdiagnosis we performed routine staining with haematoxylon and eosin for histological examination, and according to May-Grünwald-Giemsa for cytological smears, in combination with specific copper staining with rubeanic acid. Additionally, at least four different cytological smears were analysed for each dog. As the copper status of the dogs was evaluated in different ways and the results for each dog were in agreement, we consider that the phenotypic diagnoses were reliable.

The lengths of the PCR products in our study were identical to those reported by YuzbasiyanGurkan et al. (1997). The association of the $167 \mathrm{bp}$ allele with the disease implies that the gene causing copper toxicosis shows the same complete linkage disequilibrium with the marker as found in the USA, and hence that the disease is most likely identical. Different mutations in different founders could, however, depending on the time of origin, have given linkage disequilibrium with the same marker allele. Various mutations in the same gene may well be responsible for phenotypically identical diseases, as has recently been exemplified by findings with respect to canine Von Willebrand's disease (Venta et al. 1996; Brewer 1998). In another study we have investigated the population dynamics of copper toxicosis in

Table 1. PCR analysis of the copper toxicosis marker in different European Bedlington terrier populations

\begin{tabular}{|c|c|c|c|c|c|c|c|}
\hline \multirow[b]{2}{*}{ Country } & \multirow[b]{2}{*}{ No. of dogs } & \multirow[b]{2}{*}{$\%$ of population } & \multicolumn{3}{|c|}{ Genotypes no. (\%) } & \multicolumn{2}{|c|}{ Allele frequencies } \\
\hline & & & $167 / 167$ & $163 / 167$ & $163 / 163$ & 167 & 163 \\
\hline The Netherlands & 195 & 90 & $22(11)$ & $84(43)$ & $89(46)$ & 33 & 67 \\
\hline Germany & 51 & 50 & $4(9)$ & $19(43)$ & $21(48)$ & 57 & 43 \\
\hline Belgium & 44 & $10-15$ & $17(33)$ & $24(47)$ & $10(20)$ & 31 & 69 \\
\hline
\end{tabular}

The genotypes are given as the allele lengths in bp. The proportions of the total studbook populations were estimated by the number of dogs analysed and the total number of dogs in the countries of origin. 
193

Diagnostic value of a microsatellite DNA marker for copper toxicosis the Dutch Bedlington terriers (Ubbink et al. unpublished). Using epidemiological techniques (Ubbink et al. 1998), we have searched nine-generation computerized pedigrees for founders of copper toxicosis in this population. These techniques usually identify a small number of ancient founding animals of an inherited disease in a population, but for the Dutch Bedlington terriers no founders could be identified and the risk for disease was quite evenly distributed over different familial clusters. This indicates that the disease was presumably already highly prevalent before the settlement of the population in The Netherlands. It is likely that the same applies to other populations, and that one old mutation has spread from the country of origin, England, and that the genetic basis of the disease in this breed is identical throughout the world.

In the dogs examined in the present study the marker allele was not only in tight linkage disequilibrium with the disease allele, but it was also maximally informative, since it occurred only in dogs associated with the disease. The complete agreement of the predictions from the marker with the genotypes inferred from histo- and cytochemistry for copper levels in the liver copper makes the marker test suitable for routine diagnosis. With the number of dogs studied, a 100\% agreement implies a predictive accuracy of the test of at least $95 \%$. The tight linkage disequilibrium between the disease alleles and the marker alleles suggests that the disease gene may be physically very close to the microsatellite locus. Thus, we are attempting to identify the disease gene by positional cloning.

Using the DNA marker for analysis of the population, we have investigated nearly the complete Bedlington terrier population of The Netherlands, a considerable part of the Belgian population, and part of the German dogs. Analyzing the pedigrees, we found that these dogs in fact constituted one large population, and that there were many links with Bedlington terriers in other countries in Western Europe, and in Britain. It is therefore not surprising that our results in continental dogs are in agreement with the recent findings from England by Holmes et al. (1998). We have found an overall allele frequency of the $167 \mathrm{bp}$ allele, which is associated with the disease, of 0.37 , which is very similar to the frequency in the USA of $0 \cdot 39$ (Yuzbasiyan-Gurkan et al. 1997). The Dutch and German subpopulations had relatively lower frequencies of the allele associated with copper toxicosis than Belgian Bedlingtons. This may be due to the fact that breeders of dogs in The
Netherlands and the western part of Germany have had their dogs examined by liver biopsies for 15-20 years, resulting in a breeding policy against the disease and a reduction of the marker allele, whereas the Belgian breeders did not. However, this phenotypic selection does not allow the elimination of carriers. We conclude that the DNA marker test developed by Yuzbasiyan-Gurkan et al. can be used to control this genetic disease in European Bedlington terriers.

\section{Acknowledgements}

The authors would like to acknowledge Professor B.A. van Oost for technical advise and critical reading of the manuscript, and the Dutch Ministry of Agriculture, Fishery, and Environment for supporting this study with a generous grant. The Dutch Kennel Club is gratefully acknowledged for the grant by which Dr Ubbink has been able to perform his $\mathrm{PhD}$ studies over the past four years, part of which is reported in the present study. The Van Haeringen laboratory in Wageningen, The Netherlands, is acknowledged for analysing the most recent samples used in this study. Finally, the cooperation of the Dutch Bedlington terrier Club was highly appreciated.

\section{References}

Brewer G.J. (1998) Von Willebrand disease DNA testing. Canine Practice 23, 27-8.

Brewer G.J., Dick R.D., Schall W. et al. (1992) Use of zinc acetate to treat copper toxicosis in dogs. Journal of the American Veterinary Medical Association 201, 564-8.

Eriksson J. (1983) Copper toxicosis in Bedlington terriers. Acta Veterinaria Scandinavica 24, 148-52.

Hardy R.M., Stevens J.B. \& Stowe C.M. (1975) Chronic progressive hepatitis in Bedlington terriers associated with elevated liver copper concentrations. Minnesota Veterinarian 15, 13-24.

Herrtage M.E., Seymour C.A. \& White R.A. (1987) Inherited copper toxicosis in the Bedlington terriors: the prevalence in asymptomatic dogs. Journal of Small Animal Practice 28, 1141-51.

Holmes N.G., Herrtage M.E., Ryder E.J. \& Binns M.M. (1998) DNA marker C04107 for copper toxicosis in a population of Bedlington terriers in the United Kingdom. Veterinary Record 142, 351-2.

Johnson G.F., Gilbertson S.R., Goldfisher S. \& Grushof \& Sternlieb I. (1984) Cytochemical detection of copper toxicosis of Bedlington terriers. Veterinary Pathology 21, 57-60.

Johnson G.F., Sternlieb I., Tweet D.C., Grushoff P.S. \& Scheinberg I. (1980) Inheritance of copper toxicosis in Bedlington terriors. American Journal of Veterinary Research 41, 1865-6. 
194

Rothuizen, Ubbink, van Zon et al.
Miller S.A., Dykes D.D. \& Polesky K.F. (1988) A simple salting out procedure for extracting DNA from human nucleated cells. Nucleic Acids Research 16, 1215.

Owen C.A. \& Ludwig J. (1982) Inherited copper toxicosis in Bedlington terriers. American Journal of Pathology 106, 432-4.

Robertson H.M., Studdert V.P. \& Reuter R.E. (1983) Inherited copper toxicosis in Bedlington terriers. Australian Veterinary Journal 60, 235-8.

Rothuizen J. (1999) Diseases of the liver and biliary tract. In: Textbook of Small Animal Medicine (ed. by Dunn, J.), pp. 448-497 W.B. Saunders Ltd., Philadelphia, PA.

Teske E., Brinkhuis B.G.A.M., Bode P., Van den Ingh T.S.G.A.M. \& Rothuizen J. (1992) Cytological detection of copper for the diagnosis of inherited copper Toxicosis in Bedlington terriers. Veterinary Record 131, 30-2.

Thornburg L.P., Rottinghaus G., McGowan M., Kupka K., Crawford S. \& Forbes S. (1990) Hepatic copper concentrations in purebred and mixed-breed dogs. Veterinary Pathology 27, 81-8.

Twedt D.C., Hunsaker H.A. \& Allen K.G. (1988) Use of 2,3,2-tetramine as a hepatic copper chelating agent for treatment of copper hepatotoxicosis in Bedlington terriers. Journal of the American Veterinary Medical Association 193, 52-6.

Twedt D.C., Sternlieb I. \& Gilbertson S.R. (1979) Clinical, morphological, and chemical studies on copper toxicosis in Bedlington Terriers. Journal of the American Veterinary Medical Association 175, 269-75.

Ubbink G.J., Van de Broek J., Hazewinkel H.A.W. \& Rothuizen J. (1998) Risk-estimates for dichotomous disease traits using a cohort study on relatedness in purebred dog populations. Veterinary Record 142, 328-31.

Venta P.J., Yuzbasiyan-Gurkan V., Schall, S. \& Brewer G.J. (1996) Identification of a single base deletion that causes Scottish terrier Von Willebrand's disease. Proceedings Xxvth International Conference on Animal Genetics, Tours, France, 79.

Yuzbasiyan-Gurkan V., Blanton S.H., Cao Y., Ferguson P., Venta P.J. \& Brewer G.J. (1997) Linkage of a microsatellite marker to the canine copper toxicosis locus for Bedlington terriers. American Journal of Veterinary Research 58, 23-7.

Zarr J.H. (1984) Biostatistical Analysis, pp. 378- 380. Prentice Hall, Englewood Cliffs, NJ. 